\title{
Erratum
}

\section{Correction to "Fixed Points of Maps of a Nonaspherical Wedge"}

\author{
Seungwon Kim, ${ }^{1}$ Robert F. Brown, ${ }^{2}$ Adam Ericksen, ${ }^{3}$ \\ Nirattaya Khamsemanan, ${ }^{4}$ and Keith Merrill ${ }^{5}$ \\ ${ }^{1}$ Department of Mathematics, Kyungsung University, Busan 608-736, Republic of Korea \\ ${ }^{2}$ Department of Mathematics, University of California Los Angeles, Los Angeles CA 90095, USA \\ ${ }^{3}$ Department of Mathematics, University of Southern California, Los Angeles, CA 90089, USA \\ ${ }^{4}$ Sirindhorn International Institute of Technology, Thammasat University, Pathum Thani 12121, Thailand \\ ${ }^{5}$ Department of Mathematics, Brandeis University, Waltham, MA 02453, USA
}

Correspondence should be addressed to Robert F. Brown, rfb@math.ucla.edu

Received 21 June 2010; Accepted 17 July 2010

Copyright (C) 2010 Seungwon Kim et al. This is an open access article distributed under the Creative Commons Attribution License, which permits unrestricted use, distribution, and reproduction in any medium, provided the original work is properly cited.

In the original paper, it was assumed that a selfmap of $X=P \vee C$, the wedge of a real projective space $P$ and a circle $C$, is homotopic to a map that takes $P$ to itself. An example is presented of a selfmap of $X$ that fails to have this property. However, all the results of the paper are correct for maps of the pair $(X, P)$.

Let $X=P \vee C$ be the wedge of the real projective plane $P$ and the circle $C$. As the example below demonstrates, the statement on page 3 of [1] "Given a map $f: X \rightarrow X$ we may deform $f$ by a homotopy so that $f_{P}$, its restriction to $P$, maps $P$ to itself." is incorrect. If, instead of an arbitrary self-map of $X$, we consider a map of pairs $f:(X, P) \rightarrow(X, P)$, the map can be put in the standard form defined on that page and then all the results of the paper are correct for such maps of pairs.

To describe the example, represent points $x$ of the unit 2-sphere $S^{2}$ by spherical coordinates $x=(r=1, \theta, \phi)$ where $r$ denotes the radius, $\theta$ the elevation and $\phi$ the azimuth. Let $S^{2}=D_{+}^{2} \cup A_{+} \cup E \cup A_{-} \cup D_{-}^{2}$ where $x$ is in $D_{+}^{2}, A_{+}, E, A_{-}$or $D_{-}^{2}$, if $\pi / 3<\theta \leq \pi / 2, \pi / 6<$ $\theta \leq \pi / 3,-\pi / 6 \leq \theta \leq \pi / 6,-\pi / 3 \leq \theta<-\pi / 6$ or $-\pi / 2 \leq \theta<-\pi / 3$, respectively. Let $Y=S_{+}^{2} \cup I_{+} \cup S^{2} \cup I_{-} \cup S_{-}^{2}$, where $S_{ \pm}^{2}$ are the 2-spheres of radius one in $\mathbb{R}^{3}$ with centers, in cartesian coordinates, at $( \pm 2,0, \pm 2), I_{+}$denotes the points $(t, 0,1)$ for $0 \leq t \leq 2$ and $I_{-}$the points $(t, 0,-1)$ for $-2 \leq t \leq 0$. Define $\tilde{f}_{P}: S^{2} \rightarrow Y$ in the following manner. For $x=(1, \theta, \phi) \in A_{ \pm}$, let

$$
\tilde{f}_{P}(x)=\tilde{f}_{P}(1, \theta, \phi)=\left(\frac{12 \theta}{\pi}-2,0, \pm 1\right) \in \mathbb{R}^{3}
$$


in cartesian coordinates. For $(1, \theta, \phi) \in E$, set $\tilde{f}_{P}(1, \theta, \phi)=(1,3 \theta, \phi)$. Let $\rho_{ \pm}=(1, \pm \pi / 2,0) \in S^{2}$ be the poles and define $K_{ \pm}: D_{ \pm}^{2} \rightarrow S^{2}-\rho_{\mp}$ by

$$
K_{ \pm}(x)=K_{ \pm}(1, \theta, \phi)=\left(1,6 \theta \mp \frac{5 \pi}{2}, \phi\right)
$$

Returning to cartesian coordinates, define $T_{ \pm}: S^{2} \rightarrow S_{ \pm}^{2}$ by

$$
T_{ \pm}\left(x_{1}, x_{2}, x_{3}\right)=\left(x_{1} \pm 2, x_{2}, x_{3} \pm 2\right) \text {. }
$$

We complete the definition of $\tilde{f}_{P}: S^{2} \rightarrow Y$ by setting $\tilde{f}_{P}(x)=T_{ \pm} K_{ \pm}$for $x \in D_{ \pm}^{2}$. Note that $\left(\tilde{f}_{P}\right)_{*}: H_{2}\left(S^{2}, \mathbb{Z} / 2 \mathbb{Z}\right) \rightarrow H_{2}(Y, \mathbb{Z} / 2 \mathbb{Z})$ such that $\left(\tilde{f}_{P}\right)_{*}(1)=(1,1,1)$. We may embed $Y$ in the universal covering space $p: \tilde{X} \rightarrow X$ because $\tilde{X}$ is an infinite tree with a 2-sphere replacing each vertex in such a way that two edges are attached at each of two antipodal points. The embedding induces a monomorphism of homology. The map $\tilde{f}_{P}$ has been defined so that if $x,-x$ are antipodal points of $S^{2}$, then $p \tilde{f}_{P}(x)=p \tilde{f}_{P}(-x)$ and therefore $\tilde{f}_{P}$ induces a map $f_{P}: P \rightarrow X$. If $f_{P}$ were homotopic to a map $g_{P}: P \rightarrow P \subseteq X$, then the homotopy would lift to cover $g_{P}$ by a map $\tilde{g}_{P}: S^{2} \rightarrow \tilde{X}$ which sends $S^{2}$ to a single 2-sphere in $\tilde{X}$. Therefore the image of $\left(\widetilde{g}_{P}\right)_{*}: H_{2}\left(S^{2}, \mathbb{Z} / 2 \mathbb{Z}\right) \rightarrow H_{2}(\tilde{X}, \mathbb{Z} / 2 \mathbb{Z})$ would be either trivial or a single generator of $H_{2}(\tilde{X}, \mathbb{Z} / 2 \mathbb{Z})$. On the other hand, the image of $\left(\tilde{f}_{P}\right)_{*}$ in $H_{2}(\tilde{X}, \mathbb{Z} / 2 \mathbb{Z})$ is nontrivial for three generators, so no such homotopy can exist. Therefore, if $f: X \rightarrow X=P \vee C$ is a map whose restriction to $P$ is the map $f_{P}$ defined above, then it cannot be homotoped to a map that takes $P$ to itself.

\section{Acknowledgment}

The authors thank Francis Bonahon and Geoffrey Mess for their help with the example.

\section{References}

[1] S. W. Kim, R. F. Brown, A. Ericksen, N. Khamsemanan, and K. Merrill, "Fixed points of maps of a nonaspherical wedge," Fixed Point Theory and Applications, vol. 2099, Article ID 531037, 18 pages, 2009. 